\begin{tabular}{c} 
International Journal of Engineering \& Technology, $7(4.26)(2018) 274-278$ \\
International Journal of Engineering \& Technology \\
SPC \\
Website: $w$ ww. sciencepubco.com/index.php/IJET \\
Research paper \\
\hline
\end{tabular}

\title{
Statistical Method for Tracing Hydraulic Fracture Front Without Evaluation of the Normal
}

\author{
A. D. Stepanov ${ }^{1,2}$ \\ ${ }^{1}$ Peter the Great St.Petersburg Polytechnic University, Russia, 195251, St.Petersburg, Polytechnicheskaya, 29 \\ ${ }^{2}$ Institute for Problems of Mechanical Engineering, Russia, 199178, St. Petersburg, V.O., Bolshoj pr., 61 \\ *Corresponding author E-mail:a.stepanov93@yandex.ru
}

\begin{abstract}
In numerical simulation of hydraulic fracture propagation, tangent component of the fluid velocity generally considered to be neglected near the crack front. Then Reynolds transport theorem yields that the limit of the particle velocity coincides with the vector of the front propagation speed. We use this fact in combination with the Poiseuille-type equation, which implies that the particle velocity is always collinear to pressure gradient. We show that this specific feature of the hydraulic fracture problem may serve to simplify tracing the front propagation. The latter may be traced without explicit evaluation of the normal to the front, which is needed in conventional applications of the theory of propagating interfaces. Numerical experiments confirm that, despite huge errors in pressure and even greater errors in its gradient, the propagation speed, statistically averaged over a distance of a mesh size, is found quite accurate. We conclude that suggested method may simplify numerical simulation of hydraulic fractures driven by Newtonian and non-Newtonian fluids.
\end{abstract}

Keywords: hydraulic fracturing; front propagation; statistical approach

\section{Introduction}

Numerical modeling is of significance for hydraulic fracture (HF) design [13]. The mathematical formulation of the problem, used for modeling, includes the continuity equation and the movement equation of the Poiseuille type [14, 15]. Combined, they contain second spatial derivatives of the pressure. The pressure is commonly evaluated through a hypersingular integral and its values are extremely inaccurate in elements intersected by the front (tip elements). Errors in the pressure gradient, which has non-integrable singularity, are naturally even greater; and they further aggravate when evaluating the second derivatives of the pressure. For this reason, conventional schemes of HF modeling avoid evaluation of the pressure in tip elements. Instead, specially designed numerical schemes (e.g. [1]) use asymptotic equations containing the module of the velocity rather than the velocity vector itself. This leads to the necessity of the normal to the fracture front evaluation to find the velocity vector entering the discretized continuity equation. In its turn, this leads to the need for using conventional methods of the theory of propagating interfaces $[2,16]$. The latter notably complicates HF modeling. It would be of value to develop an alternative approach meeting the continuity equation and traces the front propagation without evaluation of the normal. The present work aims to develop such a method. Recall, that as mentioned in Introduction, the hypersingular operator $G$, when used in its spatially discretized form, provides rather inaccurate results for the nodal values at mesh elements intersected by the front (tip elements) and at their neighbors (ribbon elements). Therefore, the applicability of the method, which avoids explicit evaluation of the normal, depends on errors, arising when pressure gradient is used to find the particle velocity at a close vicinity of the front. We need to study these errors and their influence on the final results.

The next sections present the numerical studies performed by using three benchmark solutions. These are the solutions to (i) the Khristianovic-Geertsma-de Klerk problem (KGD problem) [5, $6,17]$, given, e.g., in the papers $[7,8]$ ); (ii) the axisymmetric problem $[9,10]$; and (iii) the truly $3 \mathrm{D}$ problem for a pay-layer between half spaces with symmetric stress contrast $[11,18]$. The numerical results show that the method avoiding explicit evaluation of the normal may be used in practical calculations. Normalization of variables was implemented modelled on the article [12]. Accordingly, variables are regarded dimensionless unless otherwise specified.

\section{Problem Formulation}

The fluid equations for the internal points of a hydraulic fracture include (i) the continuity (mass conservation) and (ii) Poiseuilletype (movement) equation. They are, respectively, (see, e.g. [1, 3]):

$\frac{\partial w}{\partial t}+\nabla \cdot(w \boldsymbol{v})+q_{l}=Q_{0}(t) \delta(x, y)$

and

$v=-\left(\frac{w^{n+1}}{\mu^{\prime}}\right)^{1 / n}|\nabla p|^{1 / n-1} \nabla p$ 
where $w$ is the opening, $\boldsymbol{v}$ is the vector of the particle velocity, $q_{l}$ is the term accounting for leak-off, $Q_{0}$ is the pumping rate, $\delta(x, y)$ is the Dirac's delta-function, $n$ is the fluid behavior index, $\mu^{\prime}=$ $2\left(2 \frac{2 n+1}{n}\right)^{n} M, M$ is its consistency index, $p$ is the fluid pressure. The pressure is connected with the opening by the elasticity hypersingular operator $G$ (e.g. [1]):

$p=G w$.

For front points, the propagation is controlled by the speed equation following from the Reynolds transport theorem [4]. It is

$\frac{d \boldsymbol{x}_{*}}{d t}=\boldsymbol{v}_{*}=\boldsymbol{n} \lim _{\boldsymbol{x} \rightarrow \boldsymbol{x}_{*}} v_{n}$,

where $\boldsymbol{v}_{*}$ is the vector of the propagation speed, $v_{n}$ is the normal component of the particle velocity, $\boldsymbol{n}$ is the normal to the fluid front Commonly, it is supposed that the component of the particle velocity, which is tangent to the front, is negligible at its vicinity. Consequently, $v_{n}=\boldsymbol{v}$, where $\boldsymbol{v}$ is the vector of the particle velocity near the front. Then (4) reads

$\boldsymbol{v}_{*}=\lim _{\boldsymbol{x} \rightarrow \boldsymbol{x}_{*}} \boldsymbol{v}$.

Equation (5) and the Poiseuille-type equation (2) imply that the propagation speed $\boldsymbol{v}_{*}$ and the normal $\boldsymbol{n}$ to the front are both collinear to pressure gradient. This non-trivial fact serves us as the basis for developing a method to trace the front without explicit evaluation of the normal, usually employed (e.g. [1]) in frames of the theory of propagating interfaces [2]. Gradient of the pressure serves us to find the direction of the propagation and to satisfy the continuity equation at points near the front without explicit evaluation of the normal.

The magnitude $v_{*}$ of the speed on the right hand side of (5) is defined by the universal asymptotic umbrella (UAU)

$w=\varphi\left(v_{*}, r\right)$,

which expresses the opening $w$ via the distancer to the front and the speed [4]. Commonly, the UAU has a simple almost monomial form $w=A_{w}\left(v_{*}\right) r^{\alpha}$, where $A_{w}$ and $\alpha$ are known functions of the speed $v_{*}$.

Since $\alpha$ changes very slowly in a quite narrow interval, inversion of (5) in the speed becomes especially simple: $v_{*}=A_{w}^{-1}\left(w / r^{\alpha}\right)$. In general, the inversion of (6) in the speed is:

$\frac{d r}{d t}=v_{*}=\varphi_{v}^{-1}(w, r)$.

In a numerical application, equation (7) defines a circle with the center at a fixed node $\boldsymbol{x}_{\boldsymbol{i}}$ under the UAU (6). A current front passes through a point $\boldsymbol{x}_{*}$ on its circumference. Since $r$ is the distance from $\boldsymbol{x}_{\boldsymbol{i}}$ to the front, the tangent to the front and the tangent to the circumference coincide at the point $\boldsymbol{x}_{*}$. This implies that the current front is an envelope of circles centered at nodal points under the UAU. Clearly, the envelope defines also the normal to the front at each its point. This serves to update the front on selected time steps. The time interval between successive updating may be quite large as the change of the normal is not significant for small time steps. The interval may be of order of time, for which the front propagates a distance of a mesh size. Hence, the continuity equation may be satisfied with sufficiently small time steps without explicit evaluation of the normal between successive updating but using the pressure gradient to define the particle velocity under the UAU. The question is: if using pressure gradient at the near-front zone is acceptable as concerns with the accuracy of numerical results?

\section{Numerical results for 1D bench-mark problem}

Consider the classical KGD problem (see, e.g. [7]). Rough mesh with merely five elements along the fracture half-length is used for numerical solution. It can be shown, that even for the rough mesh employed, the center of the ribbon element is still under the UAU. Therefore equation (7) may be confidently used to find the absolute values of the propagation speeds and, consequently, the current distances $r$ from the centers of ribbon elements to the front. This serves to conclude if the front still intersects a current tip element. If it does not, the tip element becomes a new ribbon element and the collections of tip, ribbon, internal and external elements are updated.

Of essence is that at points near the front, the particle velocity, entering the continuity equation (1), is evaluated via pressure gradient. The results of the calculations are illustrated in Figures 16 . Solid lines present the exact values of quantities; dashed curves correspond to numerical results obtained by using the pressure at tip and ribbon elements.

Fig. 1 presents graphs for the net-pressure at the center of a ribbon element within the time interval during which the front propagates the distance of a mesh size. As could be expected, particular values of the pressure drastically differ from the exact values. The errors are of order of hundred percent. The errors in pressure gradient are of the order hundreds/thousands percent (fig. 1).

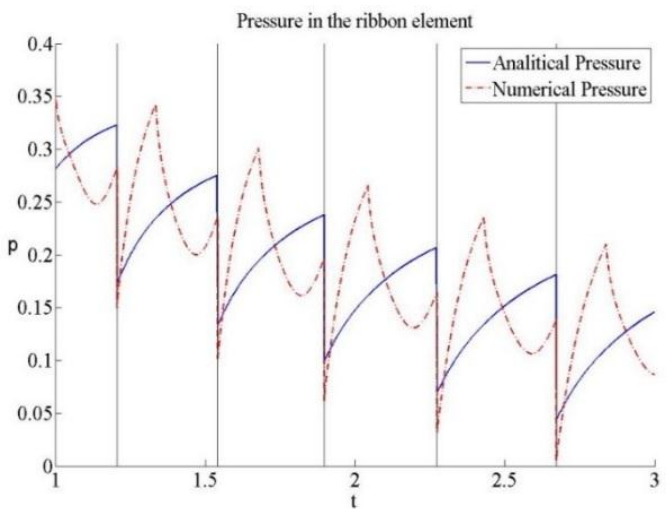

Fig. 1: Pressure at the center of a ribbon element. Vertical lines mark the instants when, due to the front propagation, a tip element becomes a new ribbon element

The particle velocity at the common side of ribbon and tip elements is calculated by applying central differences to equation (2). The velocities are given in Fig. 2. Again, it can be seen that particular values strongly differ from the exact velocities (fig. 2).

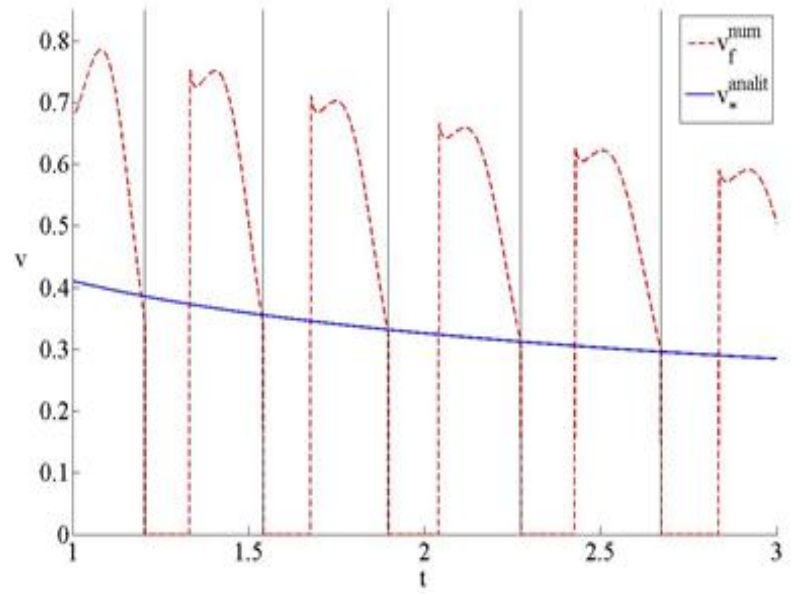

Fig. 2: Particle velocity at ribbon to tip boundary. Vertical lines mark the time when ribbon element changes.

The found particle velocity is used in the discretized conservation law (1) to find the opening at the ribbon element. The change of the 
opening in time is shown in Fig. 3. The relative error of the opening is given in Fig. 4.

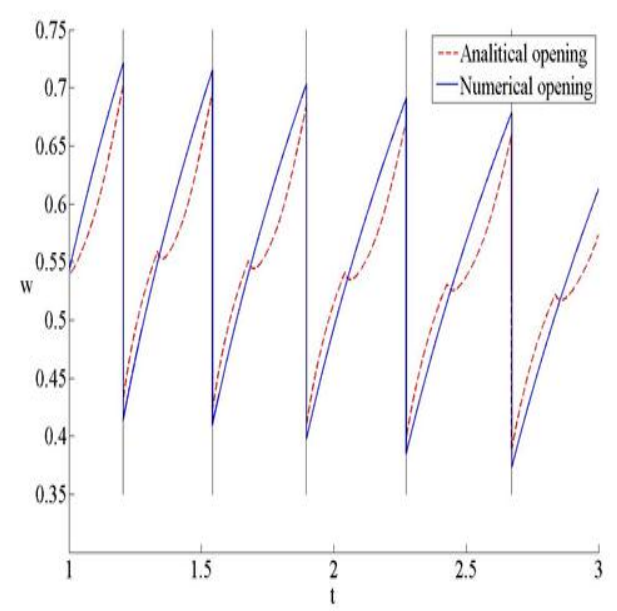

Fig. 3: Opening at the center of a ribbon-element.

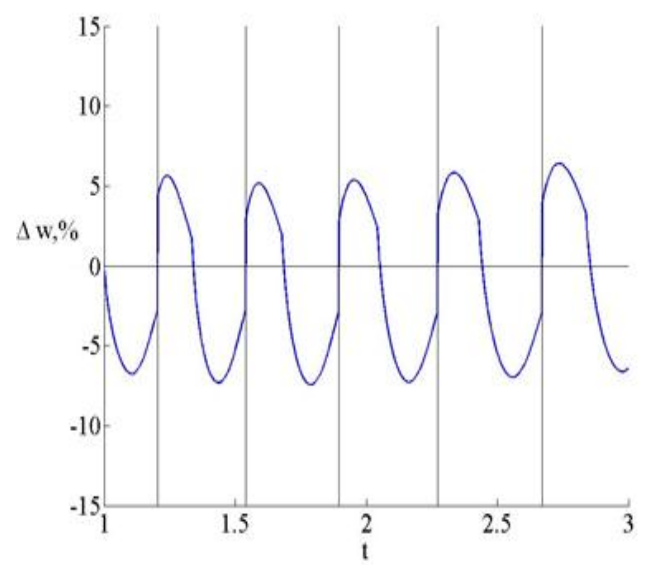

Fig. 4: The relative error of the opening at a ribbon element.

From the figures, it can be concluded that the conservation law has highly favorable smoothing effect on the numerical results. In contrast with Fig. 1 and 2 for the pressure and particle velocity near the front, Fig 3 evidently demonstrates that the errors in the opening are much less. What is of special significance, it appears that the error changes its sign near the middle of the interval, during which the front travels the distance of a mesh size. As a result, the relative average error is $1.45 \%$ only.

The accurate (in average) values of the opening yield quite accurate (in average) values of the front propagation speed. The latter, as mentioned, is found through the inversion (7) of the UAU (6). The graphs are given in Fig. 5.

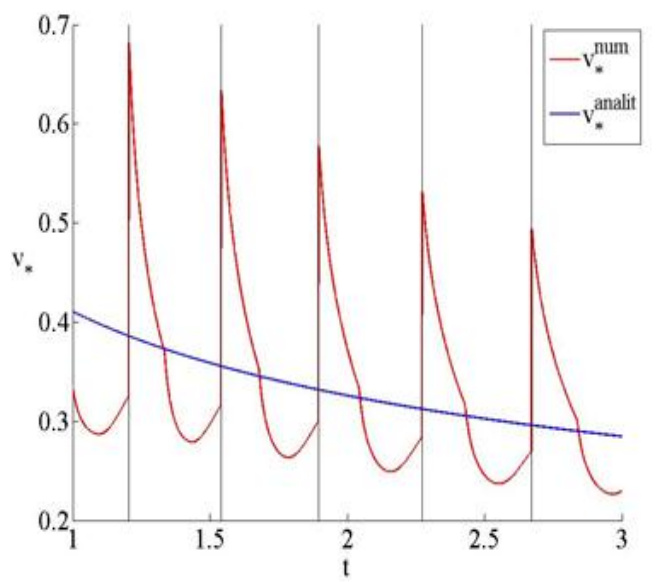

Fig. 5: Front propagation speed.

The fracture length is obtained by integration of the propagation speed. Due to the high accuracy of the average value of the speed, the accuracy of the half-length is similarly high. Its relative errors are presented in Fig. 6.

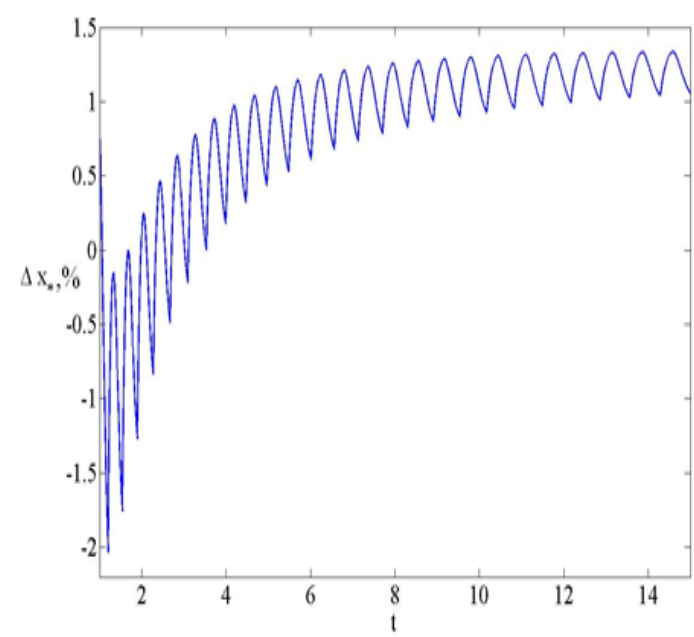

Fig. 6: Relative error of the fracture half-length.

It is also of interest to study the error at points far-away from the front. Particularly, the opening at the inlet is of special practical significance. Fig. 7 presents the relative error of this opening. It can be seen that even at the start time, the error does not exceed $2.2 \%$. It swiftly decreases with time growth.

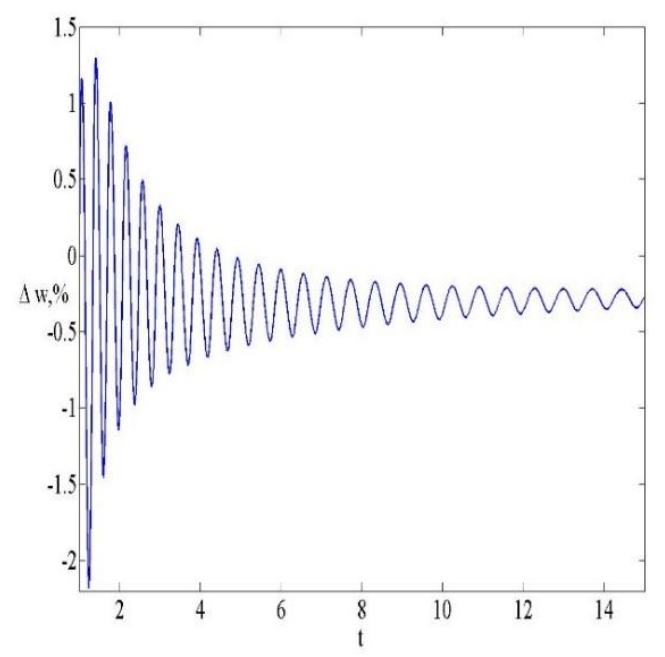

Fig. 7. Relative error of the opening at the inlet.

\section{Numerical Results for Axisymmetric Benchmark Problem}

The positive conclusion on the applicability of the method suggested to $1 \mathrm{D}$ problem, makes reasonable to extend the study to $2 \mathrm{D}$ fractures in the $3 \mathrm{D}$ space. The solution [10] to the

axisymmetric benchmark problem serves us to estimate errors. Below we present the results obtained for a Newtonian $(n=1)$ and non-Newtonian $(n=0.6)$ fluids. They are obtained for a rough mesh with merely five nodes along the initial fracture radius using explicit Euler method. Time step was taken equal to $10^{-4}$ to provide the method stability.

For the Newtonian fluid, Fig. 8, 9 present, respectively, the errors in the fracture radius and the opening at the inlet. 


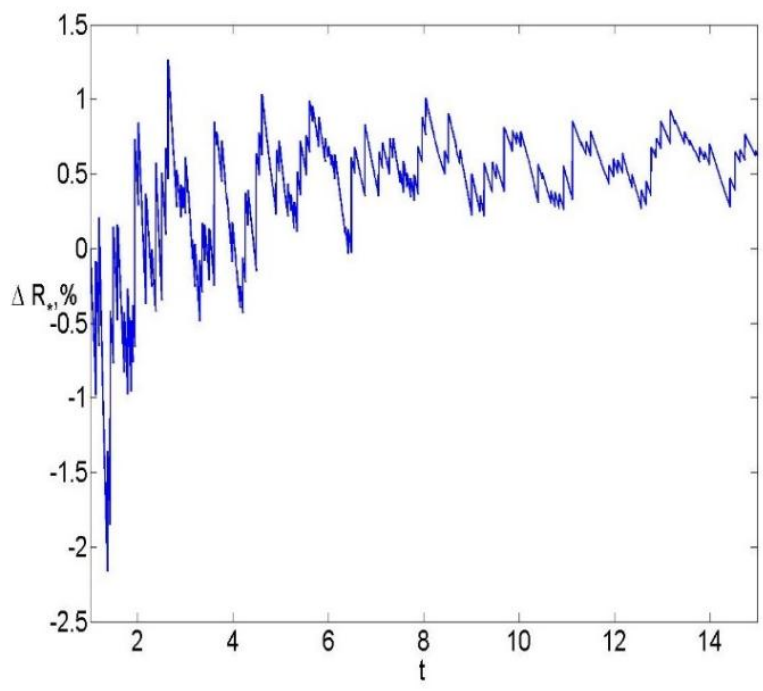

Fig. 8: Relative error of the average (along fracture circumference) fracture radius for the Newtonian fluid.

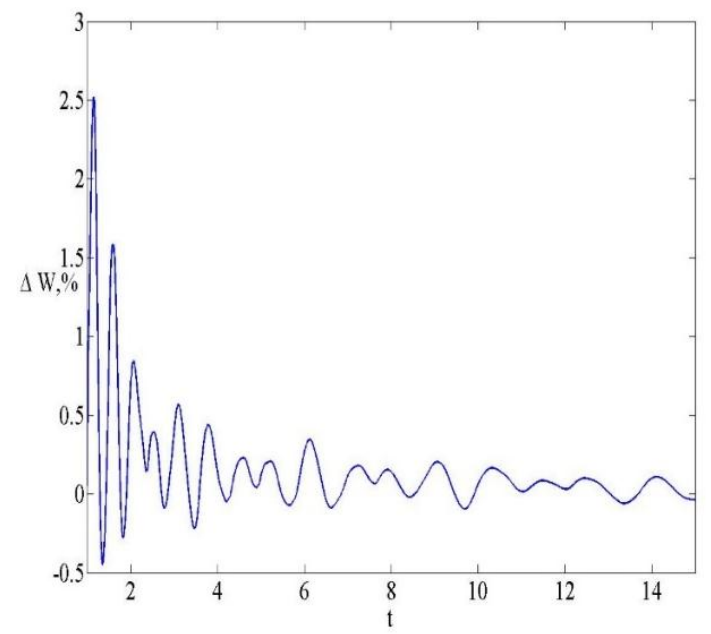

Fig. 9: Relative error of the fracture opening at the inlet for the Newtonian fluid.

Both graphs evidently show fast dying away of the error with growth of the number of mesh elements accompanying the front propagation. The maximal error does not exceed $2.5 \%$.

Similar results for the non-Newtonian fluid are presented in Fig. 10 and 11 , for the radius and the opening at the inlet, respectively.

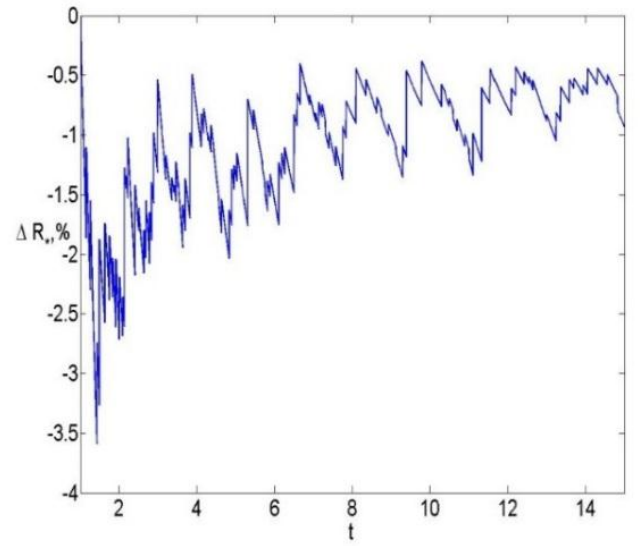

Fig. 10: Relative error of the average (along fracture circumference) fracture radius for the non-Newtonian fluid.

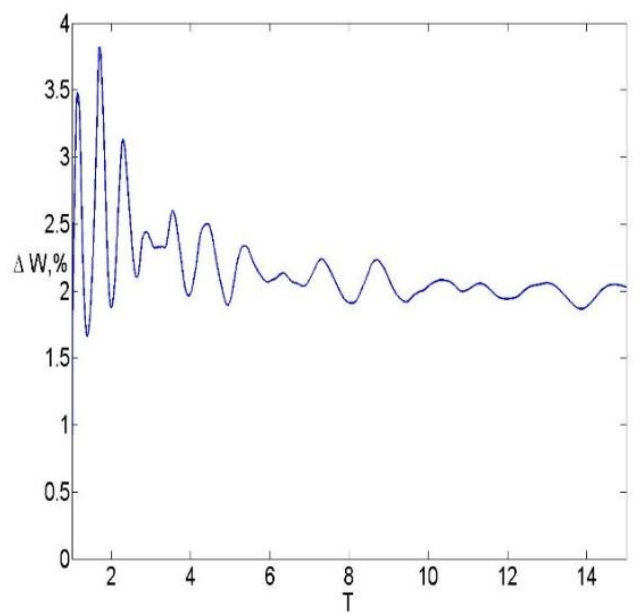

Fig. 11: Relative error of the fracture opening at the inlet for the nonNewtonian fluid.

From Fig. 10 and 11, it follows that, although the error for nonNewtonian fluids is a bit greater than that for a Newtonian fluid, it is still on a level acceptable for practical applications of the method suggested.

\section{Numerical Results for the Benchmark Problem with Stress contrast}

Consider the benchmark problem with strong stress contrast. The pay-layer of the thickness $H=0.05 \mathrm{~m}$ is located between halfspaces with the same elasticity module $E=3.3 \mathrm{GPa}$ and the same Poisson's ratio $v=0.4$. The stress contrast in the half-spaces is $\Delta \sigma=4.3 \mathrm{MPa}$. The source of the fluid with dynamic viscosity $\mu=$ $30.2 \mathrm{~Pa} \cdot \mathrm{s}$ is located at the center of the pay-layer. The pumping rate is $Q_{0}=1.7 \frac{\mathrm{mm}^{3}}{\mathrm{~s}}$. For these input data, the benchmark solution is obtained by using the implicit level set algorithm (ILSA) [ 1]; the solution is given in the paper [11]. The results of calculations by the method suggested are presented in Fig. 12 and 13 for the time instant $t=604 \mathrm{~s}$.

Fig 12 presents the opening profile at the central cross section.

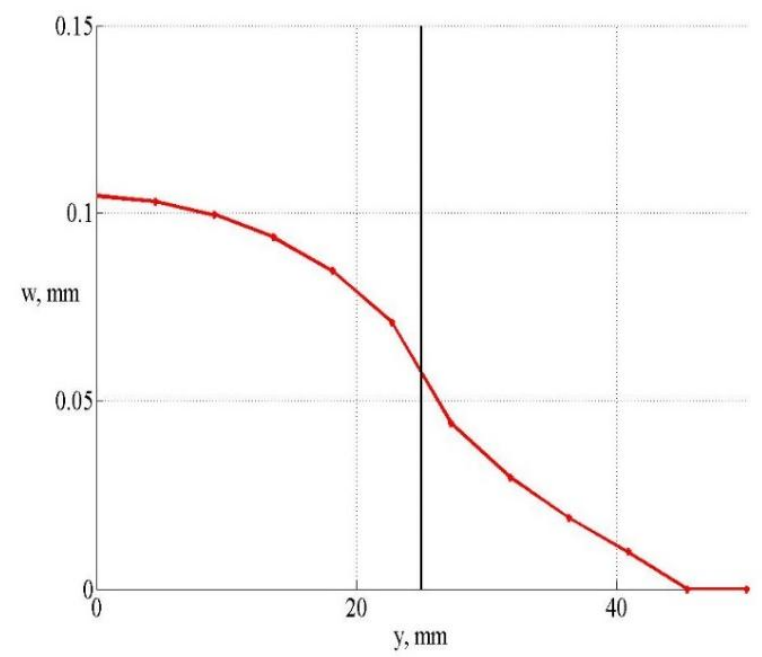

Fig. 12: The opening profile at the central cross-section. Bold line denotes the boundary of the stress-contrast.

One may find good correspondence with the results given in Fig. 7 of the paper [11]. The opening at the inlet evaluated by the method suggested is $104.6 \mathrm{~mm}$ against 105.5 obtained by the ILSA. 


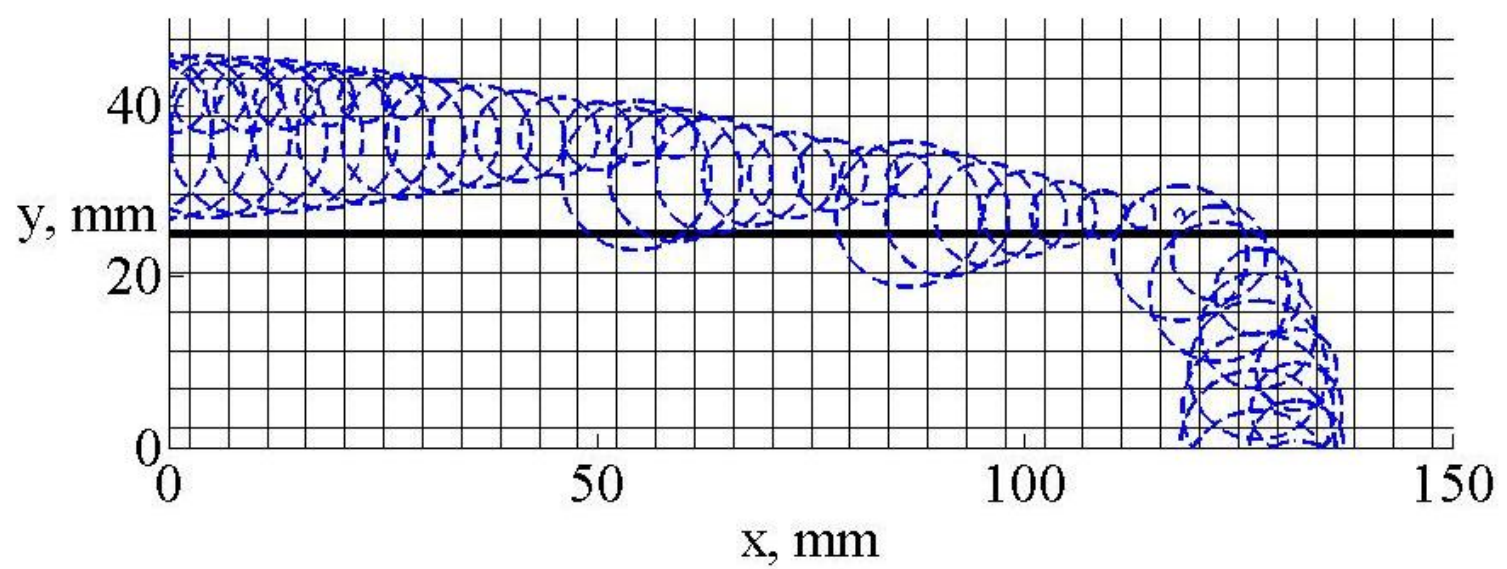

Fig. 13: Circles denote distance from the center of ribbon-elements to the facture front. Bold line denotes the boundary of the stress-contrast.

Fig 13 presents the circles defined by the speed equation (7). Its envelope corresponds to the current fracture front. The comparison of Fig. 13 with the results given in Fig. 7 of the paper [11] shows that the fracture height, evaluated by the method suggested is $45.54 \mathrm{~mm}$ against $44.57 \mathrm{~mm}$ obtained by the ILSA; the fracture lengths are, respectively, $136.88 \mathrm{~mm}$ and $136.69 \mathrm{~mm}$. The agreement confirms the applicability of the method developed.

\section{Conclusions}

The developed method simplifies tracing the front propagation by avoiding evaluation of the normal to the front at each time step. It provides acceptable numerical results for the key characteristics of a hydraulic fracture (fracture sizes and the opening): its accuracy is on the level of the most advanced code ILSA [1]. It is applicable to Newtonian, as well to non-Newtonian fluids. It may also serve to solve problems with strong stress contrast.

\section{Acknowledgements}

The work was financially supported by the Ministry of Science and Higher Education of the Russian Federation in the framework of the Federal Program "Research and Development in the Priority Areas of Development of the Russian Scientific and Technical Complex for 2014-2020", Activity 1.2., Agreement № 14.575.21.0146 of 26.09.2017. Unique project identifier: RFMEFI57517X0146.

\section{References}

[1] Peirce, A., E. Detournay, E. (2008). Comput. Methods Appl. Mech. Engng., 197, 33.

[2] Sethian, J. A. (1999). Level set methods and fast marching methods evolving interfaces in computational geometry, fluid mechanics, computer vision, and materials science (Vol. 3). Cambridge university press.

[3] Adachi, J., \& Siebrits, E. (2007). Peirce A and Desroches J. Computer simulation of hydraulic fractures. Int J Rock Mech Min Sci, 44(5), 739-57.

[4] Linkov, A. M. (2015). The particle velocity, speed equation and universal asymptotics for the efficient modelling of hydraulic fractures. Journal of Applied Mathematics and Mechanics, 79(1), 54-63.

[5] Khristianovic, S., \& Zheltov, Y. (1955, June). Formation of vertical fractures by means of highly viscous fluids. In Proc. 4th world petroleum congress, Rome (Vol. 2, pp. 579-586).
[6] Geertsma, J., \& De Klerk, F. (1969). A rapid method of predicting width and extent of hydraulically induced fractures. Journal of Petroleum Technology, 21(12), 1-571.

[7] Adachi, J. I., \& Detournay, E. (2002). Self-similar solution of a plane-strain fracture driven by a power-law fluid. International Journal for Numerical and Analytical Methods in Geomechanics, 26(6), 579-604.

[8] Linkov, A. M. (2012). On efficient simulation of hydraulic fracturing in terms of particle velocity. International Journal of Engineering Science, 52, 77-88.

[9] Savitski, A. A., \& Detournay, E. (2002). Propagation of a pennyshaped fluid-driven fracture in an impermeable rock: asymptotic solutions. International Journal of Solids and Structures, 39(26), 6311-6337.

[10] Linkov, A. (2018). On relative input of viscous shear into the elasticity equation at near-front, near-inlet and the major part of a hydraulic fracture. arXiv preprint arXiv:1808.05533.

[11] Dontsov, E. V., \& Peirce, A. P. (2016). Comparison of toughness propagation criteria for blade-like and pseudo-3D hydraulic fractures. Engineering Fracture Mechanics, 160, 238-247.

[12] Linkov, A. M. (2016). Numerical solution of plane hydrofracture problem in modified formulation under arbitrary initial conditions. Journal of Mining Science, 52(2), 265-273.

[13] Mighani, S., Lockner, D. A., Kilgore, B. D., \& Evans, B. (2018, August). Role of Fault Gouge during Interaction between Hydraulic Fracture and a Preexisting Fracture. In 52nd US Rock Mechanics/Geomechanics Symposium. American Rock Mechanics Association.

[14] Casares, L., Vincent, R., Zalvidea, D., Campillo, N., Navajas, D., Arroyo, M., \& Trepat, X. (2015). Hydraulic fracture during epithelial stretching. Nature materials, 14(3), 343.

[15] Wills, P. B., Karam, S. G., Mestayer, J. J., Ugueto, G. A., \& Lopez, J. L. (2016). U.S. Patent No. 9,347,313. Washington, DC: U.S. Patent and Trademark Office.

[16] Wu, K., \& Olson, J. E. (2016). Numerical investigation of complex hydraulic-fracture development in naturally fractured reservoirs. SPE production \& operations, 31(04), 300-309.

[17] McClure, M. W., Babazadeh, M., Shiozawa, S., \& Huang, J. (2016). Fully coupled hydromechanical simulation of hydraulic fracturing in 3D discrete-fracture networks. SPE Journal, 21(04), 1-302.

[18] Shakiba, M., \& Sepehrnoori, K. (2015, September). Using embedded discrete fracture model (EDFM) and microseismic monitoring data to characterize the complex hydraulic fracture networks. In SPE annual technical conference and exhibition. Society of Petroleum Engineers. 